\title{
Researching on the method of Rayleigh wave extraction
}

\author{
ZHU Xu-fang ${ }^{1, a^{*}, \text { YAN Bing2, }}$, \\ ${ }^{1}$ College of electronic Eng., Naval Univ. of Engineering,Wuhan,China \\ ${ }^{2}$ Dept. of Weaponry Eng., Naval Univ. of Engineering,Wuhan,China \\ a1580284687@qq.com
}

\begin{abstract}
Keywords:Seismic wave,Rayleigh wave,Seismic exploration,Polarization fliter, Covarance matrix,Noise removal
\end{abstract}

Abstract. Rayleigh wave is a secondary wave characterized by low frequency and strong energy,propagating mainly along the interface of medium and rapid attenuation of energy with increase in interface distance.The same as reflected wave and refracted wave, Rayleigh wave also contain subsurface geological information. In this paper, the concept of the Rayleigh wave, wave equation, dispersion equation, the frequency bulk characteristics and the application of the actual data are used to indicate the characteristics of Rayleigh wave and its application. According to the seismic wave field characteristics, analyzed further about the seismic wave in the form of noise and denoising method,focus on the analysis of the polarization filtering method to extract Rayleigh wave which is based on covariance matrix as the basis for the realization.

\section{Introduction}

Noise can be divided into two major categories of coherent noise and random noise[3-5]. Coherent noise is refers to the transmission frequency and velocity have certain regularity of wave, such as surface wave propagation along the ground, when the car horn, the train whistle sound waves, high voltage induction wave, etc. Random noise is chaotic vibration, such as the ground of trouble, people walk, garage and underground crushing between formation caused by vibration, etc.

\section{Seismic noise denoising method}

\section{(1) $f-x$ prediction filter.}

Suppose in frequency - coherent $(f-x)$ signal can be predicted in the spatial domain, unpredictable random noise. Can be calculated on the frequency of each section of prediction filter operator, then predict filter operator role respectively in the direction of the corresponding spatial data series, can predict coherent signal and noise suppression. Practice has proved that this method is a kind of can effectively enhance the coherent signals in seismic data continuity, suppress random noise. But because is for all of the coherent signal enhancement, and because of the high frequency band, low SNR and noise have bigger influence on the prediction operator, so that when the denoising of seismic profile of high frequency serious distortion of effective signal, reduces the fidelity of signal and SNR of section.

\section{(2) polynomial fitting denoising.}

The basic idea of this method is based on the similarity between the effective signals in space, adopt the method of multichannel related effective waves within a certain time window in phase axis position of space and time, then work out effective wave waveform within the time window, then according to the way of correlation coefficient of the signal energy distribution, thus achieving effective wave, amplitude fitting. After fitting the profile data signal-to-noise ratio are improved greatly, and no loss of high frequency components of the profile data, keep the resolution of the original signal, but also can keep the original way of relative amplitude, but using this method sometimes appear false in phase axis and earthworm phenomenon. 


\section{(3) $K-L$ transform.}

$K-L$ transform is a kind of orthogonal transform, it is the corresponding vector by sample $X$ eigenvectors $U$ corresponding to the covariance matrix of $C_{x}=X X^{T}$, namely:

$Y=U^{T} X=\sum_{i=1}^{T} x_{i} u_{i}$

Generally called $Y \quad X \quad K L$ transformation. Call $X=U Y=\sum_{i=1}^{T} y_{i} u_{i} \quad Y \quad K L$. We put each feature vector is called a principal component. By formula (1) it can be seen that $K-L$ transform generates a series of orthogonal principal component, the ith a characteristic value is the ith a measure the size of the main points the coherence of energy, and thus take the larger number of principal components to reconstruct the original signal, equivalent to the reconstruction of the original signal coherent energy. In high resolution exploration using the trail from the case, think of the adjacent channel effective components on the waveforms and energy of reflection wave has strong correlation, and random noise is not relevant, so can use this method to extract the irrelevant information, eliminate the random noise. $K-L$ transformation under the significance of variance is a best transformation. But there are some problems in practical application, due to the $K-L$ transform is using the correlation of the adjacent channel signal at the same time, so the level in phase axis denoising effect is good, but the tilt or bending in phase axis, the effect is not ideal, the other no quick $K-L$ transform algorithm, large amount of calculation.

\section{(4)based on wavelet transform high frequency random noise removal.}

Wavelet transform has good localization characteristics, which are widely used in signal processing. Wavelet transform retains the correlation of effective signals and randomness of random noise, thus in the wavelet domain to deal with the noise of seismic data. Has now developed a variety of wavelet denoising method, and in the seismic signal denoising achieved ideal effect.

\section{(5) vector decomposition denoising.}

Vector decomposition method is through the compression is not related to weight to increase the relevance of the adjacent channel signal, it uses multichannel record, according to the correlation of signal and random noise signal in the direction of statistics, utmost ground to suppress noise.Can distinguish between each other in Angle domain average recorded by multichannel signal vector and by the noise from the formation of a signal noise vectors, so the method is a kind of angular filtering. This method does not destroy the relative on the transverse and longitudinal relationship between amplitude, adapted to the in amplitude and meet the requirements of some special processing.This approach assumes that the adjacent channel signal has the similarity, so it is suitable for prestack record, also applies to after stack section, but generally used in the pile of post-processing.

\section{Extracting Rayleigh wave seismic waves}

By seismometers to receive the echo signal of composition is complex, both Rayleigh wave signal, and longitudinal wave and transverse wave signal, the particle vibration path is a vector space curve, so the seismic exploration technology to solve problems is to put the Rayleigh wave signal from seismic geophone receiving are extracted from the original signal. According to the Rayleigh wave characteristics along the ellipse motion of a particle, can adopt the method of polarization filtering, removal of longitudinal wave and transverse wave and background interference.

Polarization filtering[6-8] is also called the spatial filtering, is improve the signal-to-noise ratio in multiwave exploration and separation of the longitudinal and transverse wave a kind of important processing technology, mainly by using various wave polarization properties differences to achieve separation of longitudinal and transverse wave, filter out the purpose of interference wave.

Covariance matrix method used in the filter is a time-varying operator, the three-component seismic record some time interval calculating covariance matrix quadratic form, the result is an 
ellipsoid. The half shaft ratio reflects the linear energy orientation degree and the polarization direction. Using polarization filter can be divided into different polarization in the shape of a wave.

As shown in figure 3-1 for the particle in a three-dimensional space polarization track diagram. Its trajectory in three-dimensional space is the vibration of the ellipsoid, in $2 \mathrm{~d}$ space is an ellipse, with the index ellipsoid can describe its shape, define index ellipsoid $e_{1}=b / a, e_{2}=c / a$, in which $a$ semi-major axis for ellipsoid, $b$ for short half shaft, ${ }^{c}$ for short time half shaft. When $a=b=c, \mathrm{a}$ particle trajectory for vibration round sphere; When $b=c=0, e_{1}=e_{2}=0$, the particle trajectory of linear vibration. Long axis direction of the ellipsoid as the main direction of particle vibration.

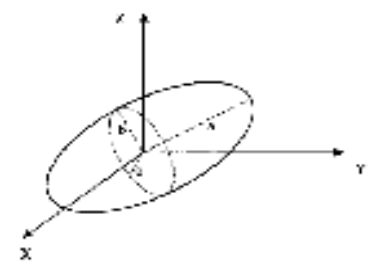

Fig. (1). particle in a three-dimensional space polarization track diagram

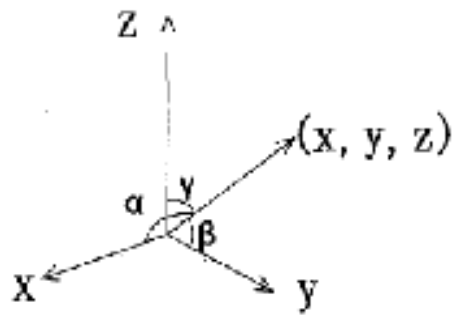

Fig. (2). main polarization direction and coordinate the relationship between the X, Y, Z

\section{(1) the covariance matrix.}

Particle trajectory can be regard as a batch of sample points on the space of random point set, so that you can use covariance matrix, using statistical methods in these space points and the polarization direction, says the quality of polarization and can use the parameters, the parameters used in filter design. In terms of three-component seismic records, select a time period is a time window as the research scope, the selection of $N$ in the three-component record sample time window $\left(\mathrm{T}_{1}, \mathrm{~T}_{2}\right)$, three component values of each point $X_{i}, Y_{i}, Z_{i}$, within the time window $\left(\mathrm{T}_{1}, \mathrm{~T}_{2}\right)$, of the mean is respectively:

$$
m_{x}=\frac{1}{N} \sum_{t=N_{1}}^{N_{2}} x_{i}, \quad m_{y}=\frac{1}{N} \sum_{t=N_{1}}^{N_{2}} y_{i}, \quad m_{z}=\frac{1}{N} \sum_{t=N_{1}}^{N_{2}} z_{i}
$$

Among them, $\left(\mathrm{N}_{2}-\mathrm{N}_{1}\right) \Delta t=T_{2}-T_{1}, \Delta t$ is the sampling rate, $N=N_{2}-N_{1}+1$.

Then using the statistical method, in view of all of the sample point data to the window average for each component within the time window, and then constructed a collaborators variance matrix. In this way, can write covariance matrix

$$
\begin{array}{r}
M_{c}=\frac{1}{N}\left[\begin{array}{ccc}
\sum A^{2} & \sum A B & \sum A C \\
\sum B A & \sum B^{2} & \sum B C \\
\sum C A & \sum C B & \sum C^{2}
\end{array}\right] \\
A=\left(\mathrm{x}_{i}-\mathrm{m}_{x}\right) ; \quad B=\left(\mathrm{y}_{i}-\mathrm{m}_{y}\right) ; \quad C=\left(\mathrm{z}_{i}-\mathrm{m}_{z}\right)
\end{array}
$$

Finally by solving matrix, the eigenvalue and eigenvector, calculate the polarization direction, constructs the modulation function. Then pair each sample point data into the Windows, multiplied by the modulation function is completed the polarization analysis respectively[10-12].

If it is linearly polarized wave, only a non-zero covariance matrix eigenvalue and the corresponding eigenvector represents the polarization direction of wave; When the wave is polarized in a plane, there are two nonzero eigenvalues and the corresponding two feature vector to determine 
the plane. In most cases, you can get three characteristic value $\lambda_{1} 、 \lambda_{2} 、 \lambda_{3}\left(\lambda_{1} \geq \lambda_{2} \geq \lambda_{3}\right)$ (eigenvalues of the matrix according to the characteristics of the equation), by the characteristic value can be set for the particle trajectory ellipsoid of short, medium and long axis, the corresponding eigenvectors can determine the direction of the axis, the biggest characteristics of the $\lambda_{1}$ value of the corresponding vector $V\left(\mathrm{~V}_{x}, \mathrm{~V}_{y}, \mathrm{~V}_{z}\right)$ feature vector is given priority to. In three characteristic value, the signal energy is concentrated in the $\lambda_{1}$ (i.e., the largest eigenvalue).

\section{(2) polarization coefficient.}

Fom the covariance matrix can be work out three characteristic values $\lambda_{1}, \lambda_{2}$ and $\lambda_{3}\left(\lambda_{1} \geq \lambda_{2} \geq \lambda_{3}\right)$. Ellipsoid are defined with the characteristic value of short, long and short time half shaft (Samson, 1977, Cliet and Dubesset, 1977), respectively:

$a=m \sqrt{\lambda_{1}}, \quad b=m \sqrt{\lambda_{2}}, \quad c=m \sqrt{\lambda_{3}}$

One $m$ is similar to $\sqrt{3}$ factors.

Total polarization coefficient was defined(Samson, 1977)

$$
T=\frac{\left(1-\mathrm{e}_{21}^{2}\right)^{2}+\left(1-\mathrm{e}_{31}^{2}\right)^{2}+\left(\mathrm{e}_{21}^{2}-\mathrm{e}_{21}^{2}\right)^{2}}{2\left(1+\mathrm{e}_{21}^{2}+\mathrm{e}_{31}^{2}\right)^{2}}
$$

$e_{21}=\sqrt{\lambda_{2} / \lambda_{1}}$ as the main index ellipsoid; $e_{31}=\sqrt{\lambda_{3} / \lambda_{1}}$ for index ellipsoid.

Polarization coefficient between 0 and 1 . When the particle linear polarization, polarization coefficient is 1 ; When ball polarization, polarization coefficient is 0 .

\section{(3)determination of polarization direction.}

Covariance matrix of the largest eigenvalue $\lambda_{1}$ corresponds to the main characteristics of vector $V$ in the cartesian coordinate system $(\mathrm{X}, \mathrm{Y}, \mathrm{Z})$ determined by three scalar coordinates $\left(\mathrm{V}_{x}, \mathrm{~V}_{y}, \mathrm{~V}_{z}\right)$ only. And the main characteristics of vector $V$ in the direction of the space is by its three direction cosine $\cos \alpha(\mathrm{t}), \cos \beta(\mathrm{t})$ and $\cos \gamma(\mathrm{t})$, namely, give priority to the polarization direction $\alpha(\mathrm{t}) 、 \beta(\mathrm{t}) 、 \gamma(\mathrm{t})$ and axis $X 、 Y 、 Z$ the Angle. According to the relation of $V_{x}^{2}+V_{y}^{2}+V_{z}^{2}=1$ and the corresponding spatial geometric relationship, can prove that the main characteristics of three direction cosine vector $V$ is equal to its three scalar coordinates, namely:

$$
\left\{\begin{array}{l}
\cos \alpha(\mathrm{t})=\mathrm{V}_{x} \\
\cos \beta(\mathrm{t})=\mathrm{V}_{y} \\
\cos \gamma(\mathrm{t})=\mathrm{V}_{z}
\end{array}\right.
$$

This also proves that the main characteristic vector to characterize the main direction of the particle polarization.

Benhama (1988), and other design which has the function of spatial orientation filter polarization filter, introducing modulation function $f(\mathrm{t})$ :

$$
f(\mathrm{t})=\mathrm{T}^{p}(\mathrm{t}) \cos ^{q} \theta(\mathrm{t})
$$

In type, $T(\mathrm{t})$ is polarization coefficient, $\theta(\mathrm{t})$ for the selected filter the Angle between the axis and the main characteristic vector $V(\mathrm{t}), \theta(\mathrm{t})$ for $\cos \alpha(\mathrm{t}), \cos \beta(t)$ or $\cos \gamma(t)$, index of $\mathrm{p}$ is weighted value of the degree of polarization, $\mathrm{q}$ is the polarization direction of the weighted value, usually $\mathrm{p}$ value range is 0 to 2 , q value in the range of 0 to 4 .

\section{Conclusion.}

Paper introduces the features of seismic wave field and the main types of noise in seismic signal, and according to the characteristics of different noise denoising methods commonly used currently in use are introduced, the advantages and disadvantages of different methods were analyzed. 
Thesis aimed at the problem of how to extract the Rayleigh wave, discusses the method of polarization filter based on covariance matrix. Its difficulty lies in estimating of the signal period, through the calculation within the time window is greater than or equal to the signal cycle integral polarization parameters, based on time window that signal cycle unit, maintain the integrity of the same waveform, based on the polarization parameters pair window within the sampling value multiplied on the weighted coefficient, make good polarity, polarization direction and filtering in the same direction of wave get relative to strengthen; Instead, polarity, polarization direction and the filtering direction vary widely waveform is relatively weak, so after filtering processing field value can still be as input values of filtering again, and can be used for further analysis of the polarization.

\section{Reference}

[1] Tang-dai xia, yong-sheng hu, shun-qun Yang, etc. Rayleigh potter road structure and dynamic response analysis. Journal of zhengzhou university of technology, 2000, 21 (1) : $19 \sim 22$.

[2] pei Jiang Yun, shu-min Chen, Liu Zhenkuan, wang. Near-surface low-velocity layer parameters inversion, geophysics, 2001, (1) : $89 \sim 92$.

[3] Backus. J G, Glfer. E. Guang-ming wang, floor of the sea. Geophysical data analysis [M]. Geological publishing house, Beijing 1988.

[4] Zhang Junhua, Lv Ning Tian Lianyu, Liu Wenzhi, Zhong Lei. Seismic data denoising method comprehensive review [J]. Progress in geophysics, Vol. 21, No. 2002 (6) : $546 \sim 553$.

[5] Wen Li, zheng-shi liu, GeYun built. Several methods of wavelet denoising [J]. Journal of hefei university of technology (natural science edition), 2002, 25 (2) : $167 \sim 172$.

[6] yu-bao zhao. The application of wavelet transform in seismic signal denoising research [D]. Master thesis, central south university, 2005.

[7] li-jun huang. Introduction to the noise in the seismic signal and remove methods [J]. Journal of Inner Mongolia petrochemical, No. 42200, $31 \sim 34$.

[8]QuanXiLong. the seismic signal identification and localization of research [D]. Hefei: anhui university, 2010. 